\title{
f-Biharmonic and Bi-f-Harmonic Magnetic Curves in Three-Dimensional Normal Almost Paracontact Metric Manifolds
}

\author{
Şerife Nur Bozdağ* and Feyza Esra Erdoğan
}

(Communicated by Marian Ioan Munteanu)

\begin{abstract}
In this paper, we study f-harmonic, f-biharmonic, bi-f-harmonic and f-biminimal non-null magnetic curves in three-dimensional normal almost paracontact metric manifolds. We determine necessary and sufficient conditions for these properties of a non-null magnetic curve. Besides, we obtain absence theorems.
\end{abstract}

Keywords: Magnetic curves, normal almost paracontact metric manifolds, f-harmonic curves, f-biharmonic curves, bi-f-harmonic curves, f-biminimal curves.

AMS Subject Classification (2020): Primary: 53C25 ; Secondary: 53C43; 58E20.

\section{Introduction}

The Serret-Frenet vectors of a charged particle are affected by a magnetic field when this charged particle entered into this area. Then with this effect, a force called Lorentz force becomes exposed and so this charged particle begin to trace an orbit called magnetic curve. The trajectories of charged particles moving on a Riemannian manifold under the action of a magnetic field are defined as magnetic curves, in [14]. The interest in magnetic fields and their corresponding magnetic curves on different manifolds is due to the fact that these concepts are one of the most important study subjects between differential geometry and physics. In the recent past, popularity of magnetic curves has increased and some of recent studies can be summarized as follows. In $[5,9,10]$, Munteanu et. al. studied Killing magnetic curves in Minkowski 3-Space and three-dimensional almost paracontact manifolds. Also they obtained magnetic curves corresponding to Killing magnetic fields in $E^{3}$. In [4], Calin et. al. studied magnetic curves in three-dimensional quasi-para-Sasakian geometry. In [24], unlike previous studies, Perktaş et al. studied biharmonicity and biminimality conditions of a non-null magnetic curve for the first time in 2018. They obtained biharmonicity and biminimality conditions of non-null magnetic curves in three-dimensional normal almost paracontact metric manifold. In this paper, we study the magnetic curves on the maps, which are briefly mentioned below, unlike the studies done so far.

Harmonic maps between Riemannian manifolds were first defined by Eells and Sampson, in [11, 12]. These maps have comprehensive field of study due to their wide applications in mathematics, engineering and physics.

Biharmonic functions, a pretty old and important subject, were first introduced by Airy and Maxwell in 1862 to identify a mathematical model of elasticity. Then biharmonic functions on Riemannian manifolds were examined by Sario et al., in [28] and by Caddeo in [3]. Eells and Sampson studied not only harmonic maps, but also biharmonic maps between the Riemannian manifolds by generalizing harmonic maps, in [11]. In recent years, the interest in biharmonic maps have two aspects; one of it, is differential geometrical aspect; classification results and constructing examples, the other one, is analytical aspect; partial differential equations, (see $[6,29,30,16])$. For some recent articles of biharmonic maps, see [24, 21, 26].

f-harmonic maps between Riemannian manifolds were defined by Lichnerowicz in 1970 and then studied 
by Lemaire and Eells, in [12]. The fact that, $\mathrm{f}$-harmonic maps have physical meaning as solutions of continuous spin systems and inhomogenous Heisenberg spin systems makes them interesting, in [2].

There is a strong relation between biharmonic and f-harmonic maps and these relation summarized in two ways by Perktaş et.al., in [25]. The first one, by extending the bienergy functional to the bi-f-energy functional, obtaning a new harmonic map called as bi-f-harmonic map which are critical points of bi-f-energy functional. The second one, by extending the f-energy functional to the f-bienergy functional, obtaning a new harmonic map called as f-biharmonic map which are critical points of f-bienergy functional, for more information see $[34,23]$.

f-biharmonic maps, which is generalization of biharmonic maps, and f-biharmonic maps between Riemannian manifolds are defined by $\mathrm{Lu}$, in $[17,18]$. Moreover, Ou obtained f-biharmonic submanifolds and maps, in [22]. He also gave complete classification of f-biharmonic curves in three-dimensional Euclidean space and characterization of f-biharmonic curves in n-dimensional space forms.

In [23], Ouakkas et. al. introduced bi-f-harmonic maps as a generalization of biharmonic and f-harmonic maps. Besides, Roth defined a non-f-harmonic, f-biharmonic map called as proper f-biharmonic map, in [27]. It should be noted that, there is not any link between the notion of f-biharmonic and bi-f-harmonic maps. In [25], Perktaş et. al. derived the bi-f-harmonic equation for curves in Riemannian manifolds and discuss the particular cases of the Euclidean space, unit sphere and hyperbolic space.

Biminimal curves in a Riemannian manifold are obtained by Loubeau and Montaldo, in [19].

Finally, Karaca and Özgür defined f-biminimal immersions and they handled f-biminimal curves in a Riemannian manifold, in [13].

Motivated by these studies in this paper, first we give basic notions which will be needed in following sections. In section 3, we remind the definition, tension and bitension fields of a magnetic curve. In section 4 , we investigate the f-harmonicity condition of a non-null magnetic curve in three-dimensional normal almost paracontact metric manifold and we give an absence theorem. In section 5, we get f-biharmonicity conditions of a non-null magnetic curve in three-dimensional normal almost paracontact metric manifold and we determine this condition in different cases such as paracosymplectic, $\beta$-para-Sasakian and $\alpha$-para-Kenmotsu manifolds. In section 6, we obtain bi-f-harmonicity conditions of a non-null magnetic curve in three-dimensional normal almost paracontact metric manifold and also discuss these conditions in various manifolds. Finally in section 7 , we get f-biminimality conditions of non-null magnetic curves in three-dimensional normal almost paracontact metric manifold.

\section{Preliminaries}

In this section, we remind some definitions and propositions which will be needed throughout the paper.

Definition 2.1. Let $(N, g)$ and $(\bar{N}, \bar{g})$ be Riemannian manifolds, then if a map $\psi:(N, g) \rightarrow(\bar{N}, \bar{g})$ is a critical point of the energy functional

$$
E(\psi)=\frac{1}{2} \int_{N}|d \psi|^{2} d v_{g}
$$

then it is defined as a harmonic map, where $d v_{g}$ is the volume element of $(N, g)$. Besides, a map called as harmonic if

$$
\tau(\psi):=\operatorname{trace} \nabla d \psi=0 .
$$

Here $\tau(\psi)$ is the Euler-Lagrange equation of the energy functional $E(\psi)$, where it is the tension field of map $\psi$ and $\nabla$ is the connection induced from the Levi-Civita connection $\nabla^{\bar{N}}$ of $\bar{N}$ and the pull-back connection $\nabla^{\psi}$, $[11,13]$.

As a natural generalization of harmonic maps, biharmonic maps are defined as below.

Definition 2.2. If a map $\psi:(N, g) \rightarrow(\bar{N}, \bar{g})$ is a critical point, for all variations, of the bienergy functional

$$
E_{2}(\psi)=\frac{1}{2} \int_{N}|\tau(\psi)|^{2} d v_{g}
$$

then it is defined as a biharmonic map.

For $\tau_{2}(\psi)$ bitension field of map $\psi$, the Euler-Lagrange equation for a biharmonic map is given as;

$$
\tau_{2}(\psi)=\operatorname{trace}\left(\nabla^{\psi} \nabla^{\psi}-\nabla_{\nabla}^{\psi}\right) \tau(\psi)-\operatorname{trace}\left(R^{\bar{N}}(d \psi, \tau(\psi)) d \psi\right)=0 .
$$

www.iejgeo.com 
Here $R^{\bar{N}}$, the curvature tensor field of $\bar{N}$, is defined as

$$
R^{\bar{N}}(K, L) M=\nabla_{K}^{\bar{N}} \nabla_{L}^{\bar{N}} M-\nabla_{L}^{\bar{N}} \nabla_{K}^{\bar{N}} M-\nabla_{[K, L]}^{\bar{N}} M
$$

for any $K, L, M \in \Gamma(T \bar{N})$ and $\nabla^{\psi}$ is the pull-back connection, [11, 13].

Note that, harmonic maps are always biharmonic. Besides non-harmonic biharmonic maps are called proper biharmonic.

Definition 2.3. If a map $\psi:(N, g) \rightarrow(\bar{N}, \bar{g})$ is critical point of the f-energy functional

$$
E_{f}(\psi)=\frac{1}{2} \int_{N} f|d \psi|^{2} d v_{g}
$$

where $f \in C^{\infty}(N, \mathbb{R})$ is a positive smooth function, it is defined as a f-harmonic map.

For $\tau_{f}(\psi)$ f-tension field of the map $\psi$, the Euler-Lagrange equation for the f-harmonic map is given as;

$$
\tau_{f}(\psi)=f \tau(\psi)+d \psi(\text { gradf })=0 .
$$

Note that, f-harmonic maps are generalizations of harmonic maps, [1, 7].

Definition 2.4. If a map $\psi:(N, g) \rightarrow(\bar{N}, \bar{g})$ is critical point of the f-bienergy functional

$$
E_{2, f}(\psi)=\frac{1}{2} \int_{N} f|\tau(\psi)|^{2} d v_{g},
$$

then it is defined as f-biharmonic map. For a f-biharmonic map, the Euler-Lagrange equation is given by

$$
\tau_{2, f}(\psi)=f \tau_{2}(\psi)+\Delta f \tau(\psi)+2 \nabla_{\text {gradf }}^{\psi} \tau(\psi)=0
$$

where $\tau_{2, f}(\psi)$ is the $\mathrm{f}$-bitension field of the map $\psi$.

If $\mathrm{f}$ is a constant then the $\mathrm{f}$-biharmonic map becomes a biharmonic map, [18, 13].

Definition 2.5. If a map $\psi:(N, g) \rightarrow(\bar{N}, \bar{g})$ is critical point of the bi-f-energy functional

$$
E_{f, 2}(\psi)=\frac{1}{2} \int_{N}\left|\tau_{f}(\psi)\right|^{2} d v_{g}
$$

then it is defined as bi-f-harmonic map.

For $\tau_{f, 2}(\psi)$ bi-f-tension field of the map $\psi$, the Euler-Lagrange equation for a bi-f-harmonic map is given as

$$
\tau_{f, 2}(\psi)=\operatorname{trace}\left(\left(\nabla^{\psi} f\left(\nabla^{\psi} \tau_{f}(\psi)\right)-f \nabla_{\nabla^{N}}^{\psi} \tau_{f}(\psi)+f R^{\bar{N}}\left(\tau_{f}(\psi), d \psi\right) d \psi\right)\right)=0,[25] .
$$

Definition 2.6. If an immersion $\psi:(N, g) \rightarrow(\bar{N}, \bar{g})$ is critical point of the bienergy functional $E_{2}(\psi)$ for variations normal to the image $\psi(N) \subset \bar{N}$, with fixed energy, then it is called biminimal. Equivalently, $\psi$ is a critical point of the $\lambda$-bienergy functional,

$$
E_{2, \lambda}(\psi)=E_{2}(\psi)+\lambda E(\psi)
$$

where $\lambda \in \mathbb{R}$ is a constant. For a $\lambda$-biminimal immersion, the Euler-Lagrange equation is

$$
\left[\tau_{2, \lambda}(\psi)\right]^{\perp}=\left[\tau_{2}(\psi)\right]^{\perp}-\lambda[\tau(\psi)]^{\perp}=0
$$

where [.] ${ }^{\perp}$ denotes the normal component of $[],.[19,13]$.

Definition 2.7. If an immersion $\psi:(N, g) \rightarrow(\bar{N}, \bar{g})$ is a critical point of the f-bienergy functional $E_{2, f}(\psi)$ for variations normal to the image $\psi(N) \subset \bar{N}$, with fixed energy, then it is called f-biminimal. Equivalently, $\psi$ is a critical point of the $\lambda$-f-bienergy functional,

$$
E_{2, \lambda, f}(\psi)=E_{2, f}(\psi)+\lambda E_{f}(\psi)
$$

where $\lambda \in \mathbb{R}$ is a constant. Then an immersion is $\mathrm{f}$-biminimal if

$$
\left[\tau_{2, \lambda, f}(\psi)\right]^{\perp}=\left[\tau_{2, f}(\psi)\right]^{\perp}-\lambda\left[\tau_{f}(\psi)\right]^{\perp}=0 .
$$

If $\mathrm{f}$ is a constant then the $\mathrm{f}$-biminimal map turns into a biminimal map, [13]. 
Definition 2.8. A differentiable manifold $N^{2 n+1}$ called as almost paracontact metric manifold if following conditions are met;

$$
\varphi^{2}=I-\eta \otimes \xi, \quad \varphi \xi=0, \quad \eta(\xi)=1, \quad g(\varphi K, \varphi L)=-g(K, L)+\eta(K) \eta(L)
$$

where $\varphi$ is a tensor field type $(1,1), \xi$ is a vector field, $\eta$ is a 1 -form, $K, L \in T N, I$ is the identity endomorphism on vector fields, $g$ is a compatible metric with a given almost paracontact structure is necessarily of signature $(n+1, n)$.

In an almost paracontact metric manifold $N, \eta \circ \varphi=0$ and $\operatorname{rank}(\varphi)=2 n$. From (2.7), $g(K, \varphi L)=-g(\varphi K, L)$ and $g(K, \xi)=\eta(K)$, for any $K, L \in T N$. The fundamental 2 -form of $N$ is defined by $\Phi(K, L)=g(K, \varphi L)$.

An almost paracontact metric manifold $(N, \varphi, \xi, \eta, g)$ is said to be normal if $N(K, L)-2 d \eta(K, L) \xi=0$, where $N$ is the Nijenhuis torsion tensor of $\varphi,[15,33]$.

For a three-dimensional normal almost paracontact metric manifold where $\alpha, \beta=$ constant, the curvature tensor field equation becomes

$$
\begin{aligned}
R(K, L) M & =\left(\frac{r}{2}+2\left(\alpha^{2}+\beta^{2}\right)\right)(g(L, M) K-g(K, M) L) \\
& +g(K, M)\left(\frac{r}{2}+3\left(\alpha^{2}+\beta^{2}\right)\right) \eta(L) \xi-\left(\frac{r}{2}+3\left(\alpha^{2}+\beta^{2}\right)\right) \eta(L) \eta(M) K \\
& -g(L, M)\left(\frac{r}{2}+3\left(\alpha^{2}+\beta^{2}\right)\right) \eta(K) \xi+\left(\frac{r}{2}+3\left(\alpha^{2}+\beta^{2}\right)\right) \eta(K) \eta(M) L
\end{aligned}
$$

here $K, L, M \in T N$ and $r$ is the scalar curvature, [24].

Proposition 2.1. For a three-dimensional almost paracontact metric manifold $N$, the following conditions are mutually equivalent:

i) $N$ is normal,

ii) there exist $\alpha, \beta$ functions on $N$ such that

$$
\left(\nabla_{K} \varphi\right) L=\alpha(g(\varphi K, L) \xi-\eta(L) \varphi K)+\beta(g(K, L) \xi-\eta(L) K),
$$

iii) there exist $\alpha, \beta$ functions on $N$ such that

$$
\nabla_{K} \xi=\alpha(K-\eta(K) \xi)+\beta \varphi K .
$$

Moreover, the functions $\alpha, \beta$ realizing (2.9) as well as (2.10) are given by

$$
2 \alpha=\operatorname{trace}\left\{K \rightarrow \nabla_{K} \xi\right\}, \quad 2 \beta=\operatorname{trace}\left\{K \rightarrow \varphi \nabla_{K} \xi\right\},
$$

[32].

Definition 2.9. Let $N$ be a three-dimensional normal almost paracontact metric manifold,

- if $\alpha=\beta=0$ then $N$ is called as paracosymplectic manifold, [8]

- if $\alpha=0$ and $\beta \neq 0$ then $N$ is called as quasi-para-Sasakian manifold, [32]

- if $\alpha=0, \beta \neq 0$ and $\beta$ is constant then $N$ is called as $\beta$-para-Sasakian manifold, in particular, if $\beta=-1$ called as para-Sasakian manifold, [33]

- $\alpha \neq 0$ and $\alpha$ is constant and $\beta=0$ then $N$ is called as $\alpha$-para-Kenmotsu manifold, [31].

Definition 2.10. Let $(N, \varphi, \xi, \eta, g)$ be a three-dimensional normal almost paracontact metric manifold. The structural function $c_{\gamma}: I \rightarrow \mathbb{R}$ of the immersed curve $\gamma: I \subseteq \mathbb{R} \rightarrow N$, is the map given by

$$
c_{\gamma}(s)=g(V(s), \xi)=\eta(V(s))
$$

where $V=\frac{d \gamma}{d s}=\dot{\gamma}$. Then the curve $\gamma$ called as slant curve if $c_{\gamma}=c=$ constant and called as Legendre curve if $c=0,[4]$.

\section{Non-Null Magnetic Curves}

In this section, we remind the definitions and tension fields of a magnetic curve on a magnetic manifold and on a three-dimensional normal almost paracontact metric manifold. 
Definition 3.1. Let $\Phi$, which is a closed 2-form on $N$, be a magnetic field on $(N, g)$ (pseudo-)Riemannian manifold. Then the Lorentz force $F_{\Phi}$ of the magnetic field $\Phi$ is defined by

$$
g\left(F_{\Phi} K, L\right)=\Phi(K, L)
$$

for any $K, L \in T N$. In this case, the magnetic curve $\gamma: I \subseteq \mathbb{R} \rightarrow N$ on the magnetic manifold $(N, g, \Phi)$ is the solution of the Lorentz equation which is given by

$$
\nabla_{\dot{\gamma}} \dot{\gamma}=F_{\Phi} \dot{\gamma}
$$

Note that, constant speed magnetic curves and geodesics are magnetic curves with vanishing magnetic fields, [4, 24].

Now let remind the definition of a magnetic curve in a three-dimensional normal almost paracontact metric manifold with $\alpha, \beta=$ constant.

Definition 3.2. A smooth curve $\gamma: I \subseteq \mathbb{R} \rightarrow N$ in a three-dimensional normal almost paracontact metric manifold $(N, \varphi, \xi, \eta, g)$ is called as a magnetic curve if it satisfies the following condition

$$
\nabla_{V} V=\varphi V
$$

where $V=\dot{\gamma}$, [24].

Throughout this article, we will assume that $\gamma: I \subseteq \mathbb{R} \rightarrow N$ is a non-null and non-geodesic magnetic curve, parametrized by arclength parameter, in a three-dimensional normal almost paracontact metric manifold $(N, \varphi, \xi, \eta, g)$ with $\alpha, \beta=$ constant as well as $\{\xi, V, \varphi V\}$ is a pseudo-orthonormal Frenet frame along the curve $\gamma$ where $V=\dot{\gamma}, g(V, V)=\varepsilon_{1}= \pm 1,(\eta(V))^{2} \neq \varepsilon_{1}$.

Let remind the tension and bitension fields of a non-null magnetic curve in a pseudo-Riemannian manifold $M$. The tension and bitension fields of a non-null magnetic curve $\gamma: I \subseteq \mathbb{R} \rightarrow M$, parametrized by arclength, immersed in a pseudo-Riemannian manifold $(M, g)$, given in [24, 20], as follows;

$$
\tau(\gamma)=\nabla_{\frac{\partial}{\partial s}}^{\gamma} d \gamma\left(\frac{\partial}{\partial s}\right)=\nabla_{V} V
$$

and

$$
\tau_{2}(\gamma)=\nabla_{V}^{3} V-R\left(V, \nabla_{V} V\right) V
$$

So we can easily see that from (3.2), if

$$
\tau(\gamma)=\nabla_{V} V=0
$$

then $\gamma$ is called as a non-null magnetic harmonic curve. Besides if

$$
\tau_{2}(\gamma)=\nabla_{V}^{3} V-R\left(V, \nabla_{V} V\right) V=0
$$

then $\gamma$ is called as a non-null magnetic biharmonic curve.

Unlike the tension field given in (3.4), more calculations are required to calculate the bitension field.

Bitension field of a non-null magnetic immersed curve $\gamma$ in a three-dimensional normal almost paracontact metric manifold $N$ with $\alpha, \beta=$ constant; obtained as below in [24] with the help of differentiating (3.1) along $\gamma$ by using (2.9);

$$
\nabla_{V}^{2} V=(1-\beta \eta(V)) V-\alpha \eta(V) \varphi V+\left(\varepsilon_{1} \beta-\eta(V)\right) \xi .
$$

Then by using (2.9) and (2.10)

$$
\begin{aligned}
\nabla_{V}^{3} V & =2 \alpha \eta(V)(\beta \eta(V)-1) V \\
& +\left(2 \alpha^{2}(\eta(V))^{2}-2 \beta \eta(V)+\varepsilon_{1}\left(\beta^{2}-\alpha^{2}\right)+1\right) \varphi V \\
& +\left(3 \alpha(\eta(V))^{2}-2 \varepsilon_{1} \alpha \beta \eta(V)-\varepsilon_{1} \alpha\right) \xi .
\end{aligned}
$$

From (2.8) and (3.1)

$$
R\left(V, \nabla_{V} V\right) V=\left(\frac{r}{2}\left((\eta(V))^{2}-\varepsilon_{1}\right)+\left(\alpha^{2}+\beta^{2}\right)\left(3(\eta(V))^{2}-2 \varepsilon_{1}\right)\right) \varphi V
$$


where $r$ is the scalar curvature of $N$. So the bitension field obtained as

$$
\begin{aligned}
\tau_{2}(\gamma) & =(2 \alpha \eta(V)(\beta \eta(V)-1)) V \\
& +\left(\left(\alpha^{2}+3 \beta^{2}+\frac{r}{2}\right)\left(\varepsilon_{1}-(\eta(V))^{2}\right)-2 \beta \eta(V)+1\right) \varphi V \\
& +\left(3 \alpha(\eta(V))^{2}-2 \varepsilon_{1} \alpha \beta \eta(V)-\varepsilon_{1} \alpha\right) \xi .
\end{aligned}
$$

With the help of these solutions, we obtained f-tension field, f-bitension field, bi-f-tension field, the biminimality and f-biminimality conditions of a non-null magnetic curve in a three-dimensional normal almost paracontact metric manifold as in following sections.

\section{4. f-Harmonic Non-Null Magnetic Curves}

In this section, we derive the f-harmonicity condition for a non-null magnetic curve in a three-dimensional normal almost paracontact metric manifold. Let $\gamma: I \subseteq \mathbb{R} \rightarrow N$ be a non-null magnetic curve in a threedimensional normal almost paracontact metric manifold with $\alpha, \beta=$ constant. Then with the help of Definition 2.3 and Definition 3.2; the f-harmonicity condition obtained as below;

$$
\tau_{f}(\gamma)=f^{\prime} V+f \varphi V=0 .
$$

Via (4.1) we get following absence theorem,

Theorem 4.1. There is no f-harmonic non-null magnetic curve in a three-dimensional normal almost paracontact metric manifold with $\alpha, \beta=$ constant.

Proof. From the f-harmonicity condition given in (4.1), it is easy to see that $f^{\prime}=0$ and $f=0$. This is a contradiction with the definition of a f-harmonic curve.

\section{5. f-Biharmonic Non-Null Magnetic Curves}

In this section, we derive the f-biharmonicity condition for a non-null magnetic curve in a three-dimensional normal almost paracontact metric manifold and discuss the particular cases of paracosymplectic, $\alpha$-paraKenmotsu and $\beta$-para-Sasakian manifolds. By using equations (3.1), (3.5), (3.6) and (3.7) in the formula of f-bitension field $\tau_{2, f}(\gamma)$, we get f-biharmonicity condition as below;

$$
\begin{aligned}
\tau_{2, f}(\gamma) & =f \tau_{2}(\gamma)+(\Delta f) \tau(\gamma)+2 \nabla_{\text {gradf }}^{\gamma} \tau(\gamma) \\
& =f\left(\nabla_{V}^{3} V-R\left(V, \nabla_{V} V\right) V\right)+f^{\prime \prime} \nabla_{V} V+2 f^{\prime} \nabla_{V}^{2} V \\
& =\left[2(1-\beta \eta(V))\left(f^{\prime}-\alpha \eta(V) f\right)\right] V \\
& +\left[\left(1-\frac{r}{2}\left((\eta(V))^{2}-\varepsilon_{1}\right)-2 \beta \eta(V)+\left(\alpha^{2}+3 \beta^{2}\right)\left(\varepsilon_{1}-(\eta(V))^{2}\right)\right) f-2 \alpha \eta(V) f^{\prime}+f^{\prime \prime}\right] \varphi V \\
& +\left[\left(3 \alpha(\eta(V))^{2}-2 \varepsilon_{1} \alpha \beta \eta(V)-\varepsilon_{1} \alpha\right) f+2\left(\varepsilon_{1} \beta-\eta(V)\right) f^{\prime}\right] \xi \\
& =0 .
\end{aligned}
$$

From (5.1) we obtain following theorem;

Theorem 5.1. Let $N$ be a three-dimensional normal almost paracontact metric manifold with $\alpha, \beta=$ constant and $\gamma: I \subseteq \mathbb{R} \rightarrow N$ be a non-null magnetic curve. Then $\gamma$ is a f-biharmonic curve iff the followings holds:

$$
\left\{\begin{array}{l}
(1-\beta \eta(V))\left(f^{\prime}-\alpha \eta(V) f\right)=0, \\
\left(1-\frac{r}{2}\left((\eta(V))^{2}-\varepsilon_{1}\right)-2 \beta \eta(V)+\left(\alpha^{2}+3 \beta^{2}\right)\left(\varepsilon_{1}-(\eta(V))^{2}\right)\right) f-2 \alpha \eta(V) f^{\prime}+f^{\prime \prime}=0 \\
\left(3 \alpha(\eta(V))^{2}-2 \varepsilon_{1} \alpha \beta \eta(V)-\varepsilon_{1} \alpha\right) f+2\left(\varepsilon_{1} \beta-\eta(V)\right) f^{\prime}=0 .
\end{array}\right.
$$


After Theorem 5.1 has been obtained, we examine the following 9 cases with the help of equation (5.2).

Case I: If $1-\beta \eta(V)=0$ and $f^{\prime}-\alpha \eta(V) f=0$ then we have followings;

$$
\left\{\begin{array}{l}
\eta(V)=\frac{1}{\beta} \\
f=\text { constant } \\
\alpha=0 .
\end{array}\right.
$$

Then we obtained the following absence theorem from Case I;

Theorem 5.2. There is no f-biharmonic non-null magnetic curve on a three-dimensional normal almost paracontact metric manifold with $\alpha, \beta=$ constant where $1-\beta \eta(V)=0$ and $f^{\prime}-\alpha \eta(V) f=0$.

Case II: If $1-\beta \eta(V) \neq 0$ and $f^{\prime}-\alpha \eta(V) f=0$, then we have followings;

$$
\left\{\begin{array}{l}
\eta(V) \neq \frac{1}{\beta} \\
f=\text { constant } \\
\alpha=0 .
\end{array}\right.
$$

From Case II we get the following absence theorem;

Theorem 5.3. There is no f-biharmonic non-null magnetic curve on a three-dimensional normal almost paracontact metric manifold with $\alpha, \beta=$ constant where $1-\beta \eta(V) \neq 0$ and $f^{\prime}-\alpha \eta(V) f=0$.

Case III: If $1-\beta \eta(V)=0$ and $f^{\prime}-\alpha \eta(V) f \neq 0$, then we have followings;

$$
\left\{\begin{array}{l}
\eta(V)=\frac{1}{\beta}, \\
f=e^{\frac{3 \alpha}{2 \beta} s+c}, \\
r=\frac{2 \beta^{2} \varepsilon_{1}\left(\alpha^{2}+3 \beta^{2}\right)-8 \beta^{2}-\frac{7}{2} \alpha^{2}}{1-\varepsilon_{1} \beta^{2}} .
\end{array}\right.
$$

Then we have Corollary 5.1 from Case III;

Corollary 5.1. Let $N$ be a three-dimensional normal almost paracontact metric manifold with $\alpha, \beta=$ constant and $\gamma: I \subseteq \mathbb{R} \rightarrow N$ is a non-null slant magnetic curve. Then $\gamma$ is a f-biharmonic curve iff the function $f$ and the constant scalar curvature requals to:

$$
f(s)=e^{\frac{3 \alpha}{2 \beta} s+c}
$$

and

$$
r=\frac{2 \beta^{2} \varepsilon_{1}\left(\alpha^{2}+3 \beta^{2}\right)-8 \beta^{2}-\frac{7}{2} \alpha^{2}}{1-\varepsilon_{1} \beta^{2}}
$$

where $s \in I, 1-\beta \eta(V)=0$ and $f^{\prime}-\alpha \eta(V) f \neq 0$.

Case IV: If $\eta(V)=0$, that is to say $\gamma$ is a Legendre curve, then equation (5.2) becomes:

$$
\left\{\begin{array}{l}
f^{\prime}=0 \\
\left(\alpha^{2} \varepsilon_{1}+3 \beta^{2} \varepsilon_{1}+1+\frac{r}{2} \varepsilon_{1}\right) f=0, \\
\alpha f=0 .
\end{array}\right.
$$

We have the following results from Case IV;

Theorem 5.4. There is no f-biharmonic non-null magnetic Legendre curve in three-dimensional normal almost paracontact metric manifold with $\alpha, \beta=$ constant. 
Corollary 5.2. Let $N$ be a $\beta$-para-Sasakian manifold and $\gamma: I \subseteq \mathbb{R} \rightarrow N$ is a non-null Legendre magnetic curve. Then $\gamma$ is a biharmonic curve iff the constant scalar curvature $r$ equals to:

$$
r=-6 \beta^{2}-2 \varepsilon_{1} .
$$

Case V: If $\beta=0$, that is to say $N$ is a $\alpha$-para-Kenmotsu manifold, then equation (5.2) becomes:

$$
\left\{\begin{array}{l}
f^{\prime}-\alpha \eta(V) f=0 \\
\left(1-\frac{r}{2}\left((\eta(V))^{2}-\varepsilon_{1}\right)+\alpha^{2}\left(\varepsilon_{1}-(\eta(V))^{2}\right) f-2 \alpha \eta(V) f^{\prime}+f^{\prime \prime}=0\right. \\
\left(3 \alpha(\eta(V))^{2}-\varepsilon_{1} \alpha\right) f-2 \eta(V) f^{\prime}=0 .
\end{array}\right.
$$

Here by using first and second equations of (5.7), we obtain the function $f$ as $f(s)=e^{\alpha \eta(V) s+c}$ and the scalar curvature $r$ as $r=\frac{2}{\eta(V)^{2}-\varepsilon_{1}}$. Then by using this results in third equation we get $\alpha=0$.

With the help of these information we have the following results from Case V;

Theorem 5.5. There is no f-biharmonic non-null magnetic curve in a $\alpha$-para-Kenmotsu manifold.

Corollary 5.3. Let $N$ be a paracoymplectic manifold and $\gamma: I \subseteq \mathbb{R} \rightarrow N$ is a non-null magnetic curve. Then $\gamma$ is a biharmonic curve iff the constant scalar curvature requals to:

$$
r=\frac{2}{\eta(V)^{2}-\varepsilon_{1}}
$$

Case VI: If $\alpha=0$, that is to say $N$ is a $\beta$-para-Sasakian manifold then equation (5.2) becomes:

$$
\left\{\begin{array}{l}
(1-\beta \eta(V)) f^{\prime}=0, \\
\left(1-\frac{r}{2}\left((\eta(V))^{2}-\varepsilon_{1}\right)-2 \beta \eta(V)+3 \beta^{2}\left(\varepsilon_{1}-\left(\eta(V)^{2}\right)\right) f+f^{\prime \prime}=0\right. \\
\left(\varepsilon_{1} \beta-\eta(V)\right) f^{\prime}=0 .
\end{array}\right.
$$

We have the following corollaries by using first equation of Case VI;

If $1-\beta \eta(V)=0$ and $f^{\prime} \neq 0$ then we have;

Corollary 5.4. Let $N$ be a $\beta$-para-Sasakian manifold and $\gamma: I \subseteq \mathbb{R} \rightarrow N$ is a non-null slant magnetic curve. Then $\gamma$ is a $f$-biharmonic curve iff the function $f$ and the constant scalar curvature requals to:

$$
f(s) \neq \text { constant }
$$

and

$$
r=\frac{2 \beta^{2} f^{\prime \prime}-8 \beta^{2} f+6 \beta^{4} \varepsilon_{1} f}{\left(1-\varepsilon_{1} \beta^{2}\right) f}
$$

where $s \in I$ and $1-\beta \eta(V)=0$.

If $1-\beta \eta(V) \neq 0$ and $f^{\prime}=0$ then we have;

Corollary 5.5. Let $N$ be a $\beta$-para-Sasakian manifold and $\gamma: I \subseteq \mathbb{R} \rightarrow N$ is a non-null magnetic curve. Then $\gamma$ is a biharmonic curve iff the constant scalar curvature requals to:

$$
r=\frac{2-4 \beta \eta(V)+6 \beta^{2}\left(\varepsilon_{1}-(\eta(V))^{2}\right)}{(\eta(V))^{2}-\varepsilon_{1}}
$$

where $1-\beta \eta(V) \neq 0$ and $f^{\prime}=0$.

If $1-\beta \eta(V)=0$ and $f^{\prime}=0$ then we have; 
Corollary 5.6. Let $N$ be a $\beta$-para-Sasakian manifold and $\gamma: I \subseteq \mathbb{R} \rightarrow N$ is a non-null magnetic slant curve. Then $\gamma$ is a biharmonic curve iff the constant scalar curvature $r$ equals to:

$$
r=\frac{2\left(3 \beta^{2} \varepsilon_{1}-3 \beta^{2}(\eta(V))^{2}-\beta \eta(V)+1\right)}{(\eta(V))^{2}-\varepsilon_{1}}
$$

where $1-\beta \eta(V)=0$ and $f^{\prime}=0$.

Case VII: If $\eta(V)=\beta=0$, that is to say $\gamma$ is a Legendre curve in $\alpha$-para-Kenmotsu manifold $N$ then equation (5.2) becomes:

$$
\left\{\begin{array}{l}
f^{\prime}=0, \\
\left(\alpha^{2} \varepsilon_{1}+1+\frac{r}{2} \varepsilon_{1}\right) f=0, \\
\alpha f=0 .
\end{array}\right.
$$

We have the following results from Case VII;

Theorem 5.6. There is no f-biharmonic non-null magnetic Legendre curve in paracosymplectic manifold.

Corollary 5.7. Let $N$ be a paracosymplectic manifold and $\gamma: I \subseteq \mathbb{R} \rightarrow N$ is a non-null magnetic Legendre curve. Then $\gamma$ is a biharmonic curve iff the constant scalar curvature $r$ equals to:

$$
r=-2 \varepsilon_{1} .
$$

Case VIII: If $\eta(V)=\alpha=0$, that is to say $\gamma$ is a Legendre curve in $\beta$-para-Sasakian manifold $N$ then equation (5.2) becomes:

$$
\left\{\begin{array}{l}
f^{\prime}=0, \\
\left(1+\frac{r}{2} \varepsilon_{1}+3 \beta^{2} \varepsilon_{1}\right) f+f^{\prime \prime}=0, \\
\beta f^{\prime}=0 .
\end{array}\right.
$$

We have the following corollary from Case VIII;

Corollary 5.8. Let $N$ be a $\beta$-para-Sasakian manifold and $\gamma: I \subseteq \mathbb{R} \rightarrow N$ is a non-null magnetic Legendre curve. Then $\gamma$ is a biharmonic curve iff the constant scalar curvature $r$ equals to:

$$
r=-6 \beta^{2}-2 \varepsilon_{1} .
$$

Case IX: If $\beta=\alpha=0$, that is to say $N$ is a paracosymplectic manifold, then equation (5.2) becomes:

$$
\left\{\begin{array}{l}
f^{\prime}=0, \\
\left(1+\frac{r}{2}\left(\varepsilon_{1}-(\eta(V))^{2}\right)\right) f+f^{\prime \prime}=0, \\
\eta(V) f^{\prime}=0 .
\end{array}\right.
$$

We have the following results from Case IX;

Theorem 5.7. There is no f-biharmonic non-null magnetic curve in a paracosymplectic manifold.

Corollary 5.9. Let $N$ be a paracosymplectic manifold and $\gamma: I \subseteq \mathbb{R} \rightarrow N$ is a non-null magnetic curve. Then $\gamma$ is a biharmonic curve iff the constant scalar curvature r equals to:

$$
r=\frac{2}{(\eta(V))^{2}-\varepsilon_{1}} .
$$

This Corollary 5.9 is the same with the Corollary 2 in the [24]. 


\section{Bi-f-Harmonic Non-Null Magnetic Curves}

In this section, we derive the bi-f-harmonicity condition for a non-null magnetic curve in a three-dimensional normal almost paracontact metric manifold and discuss the particular cases of paracosymplectic, $\beta$-paraSasakian and $\alpha$-para-Kenmotsu manifolds. By using equations (3.1), (3.5), (3.6) and (3.7) in the equation of bi-f-tension field $\tau_{f, 2}(\gamma)$, we get bi-f-harmonicity condition as below;

$$
\begin{aligned}
\tau_{f, 2}(\gamma)= & \operatorname{trace}\left(\nabla^{\gamma} f\left(\nabla^{\gamma} \tau_{f}(\gamma)\right)-f \nabla_{\nabla}^{\gamma} \tau_{f}(\gamma)+f R\left(\tau_{f}(\gamma), d \gamma\right) d \gamma\right) \\
= & \left(f f^{\prime \prime \prime}+f^{\prime} f^{\prime \prime}\right) V+\left(3 f f^{\prime \prime}+2\left(f^{\prime}\right)^{2}\right) \nabla_{V} V+4 f f^{\prime} \nabla_{V}^{2} V+f^{2} \nabla_{V}^{3} V+f^{2} R\left(\nabla_{V} V, V\right) V \\
= & {\left[\left(f f^{\prime \prime}\right)^{\prime}+(1-\beta \eta(V))\left(4 f f^{\prime}-2 f^{2} \alpha \eta(V)\right)\right] V } \\
+ & {\left[3 f f^{\prime \prime}+2\left(f^{\prime}\right)^{2}-4 f f^{\prime} \alpha \eta(V)+f^{2}\left(1-2 \beta \eta(V)+\frac{r}{2}\left((\eta(V))^{2}-\varepsilon_{1}\right)\right.\right.} \\
& \left.\left.+(\eta(V))^{2}\left(5 \alpha^{2}+3 \beta^{2}\right)-\varepsilon_{1}\left(\beta^{2}+3 \alpha^{2}\right)\right)\right] \varphi V \\
+ & {\left[4 f f^{\prime}\left(\varepsilon_{1} \beta-\eta(V)\right)+f^{2}\left(3 \alpha(\eta(V))^{2}-2 \varepsilon_{1} \alpha \beta \eta(V)-\varepsilon_{1} \alpha\right)\right] \xi=0 . }
\end{aligned}
$$

From equation (6.1) we obtain the following theorem;

Theorem 6.1. Let $N$ be a three-dimensional normal almost paracontact metric manifold with $\alpha, \beta=$ constant and $\gamma: I \subseteq \mathbb{R} \rightarrow N$ be a non-null magnetic curve. Then $\gamma$ is a bi-f-harmonic curve if and only if the followings holds:

$$
\left\{\begin{array}{l}
\left(f f^{\prime \prime}\right)^{\prime}+(1-\beta \eta(V))\left(4 f f^{\prime}-2 f^{2} \alpha \eta(V)\right)=0, \\
3 f f^{\prime \prime}+2\left(f^{\prime}\right)^{2}-4 f f^{\prime} \alpha \eta(V)+f^{2}\left[1-2 \beta \eta(V)+\frac{r}{2}\left((\eta(V))^{2}-\varepsilon_{1}\right)+(\eta(V))^{2}\left(5 \alpha^{2}+3 \beta^{2}\right)-\varepsilon_{1}\left(\beta^{2}+3 \alpha^{2}\right)\right]=0, \\
4 f f^{\prime}\left(\varepsilon_{1} \beta-\eta(V)\right)+f^{2}\left(3 \alpha(\eta(V))^{2}-2 \varepsilon_{1} \alpha \beta \eta(V)-\varepsilon_{1} \alpha\right)=0 .
\end{array}\right.
$$

Let's examine Theorem 6.1 in detail.

Case I: If $\eta(V)=0$, that is to say $\gamma$ is a Legendre curve, then equation (6.2) becomes:

$$
\left\{\begin{array}{l}
\left(f f^{\prime \prime}\right)^{\prime}+\left(2 f^{2}\right)^{\prime}=0 \\
3 f f^{\prime \prime}+2\left(f^{\prime}\right)^{2}+f^{2}\left(-\varepsilon_{1}\left(\beta^{2}+3 \alpha^{2}\right)+1-\frac{r}{2} \varepsilon_{1}\right)=0 \\
4 f f^{\prime} \beta-\alpha f^{2}=0
\end{array}\right.
$$

We have the following corollary from Case I;

Corollary 6.1. Let $N$ be a $\beta$-para-Sasakian manifold and $\gamma: I \subseteq \mathbb{R} \rightarrow N$ is a non-null Legendre magnetic curve. Then $\gamma$ is a bi-f-harmonic curve iff the function $f$ and the constant scalar curvature $r$ equals to:

$$
f(s)=\text { constant }
$$

and

$$
r=2\left(\varepsilon_{1}-\beta^{2}\right)
$$

where $s \in I$.

Case II: If $\beta=0$, that is to say $N$ is a $\alpha$-para-Kenmotsu manifold, then equation (6.2) becomes:

$$
\left\{\begin{array}{l}
\left(f f^{\prime \prime}\right)^{\prime}+\left(2 f^{2}\right)^{\prime}-2 f^{2} \alpha \eta(V)=0, \\
3 f f^{\prime \prime}+2\left(f^{\prime}\right)^{2}-4 f f^{\prime} \alpha \eta(V)+f^{2}\left(\alpha^{2}\left(5(\eta(V))^{2}-3 \varepsilon_{1}\right)+1+\frac{r}{2}\left((\eta(V))^{2}-\varepsilon_{1}\right)\right)=0, \\
-4 f f^{\prime} \eta(V)+f^{2}\left(3 \alpha(\eta(V))^{2}-\varepsilon_{1} \alpha\right)=0 .
\end{array}\right.
$$

We have the following corollaries from Case II; 
Corollary 6.2. Let $N$ be a $\alpha$-para-Kenmotsu manifold and $\gamma: I \subseteq \mathbb{R} \rightarrow N$ is a non-null magnetic curve. Then $\gamma$ is a bi-f-harmonic curve iff the function $f$ and the constant scalar curvature $r$ equals to:

$$
f(s)=e^{\left(\frac{3 \alpha(\eta(V))^{2}-\varepsilon_{1} \alpha}{4 \eta(V)}\right) s+c}
$$

and

$$
r=\frac{\left(10\left(\frac{3 \alpha(\eta(V))^{2}-\varepsilon_{1} \alpha}{4 \eta(V)}\right)^{2}-8\left(\frac{3 \alpha(\eta(V))^{2}-\varepsilon_{1} \alpha}{4 \eta(V)}\right) \alpha \eta(V)+10 \alpha^{2}(\eta(V))^{2}-6 \alpha^{2} \varepsilon_{1}+2\right)}{\varepsilon_{1}-(\eta(V))^{2}}
$$

where

$$
\alpha=\frac{\varepsilon_{1} \pm 24 \eta(V) \sqrt{2\left(3(\eta(V))^{4}+(\eta(V))^{2}-1\right)\left(\varepsilon_{1}-(\eta(V))^{2}\right)}}{18(\eta(V))^{2}\left(3(\eta(V))^{4}+(\eta(V))^{2}-1\right)}
$$

and $s \in I$.

Corollary 6.3. Let $N$ be a paracosymplectic manifold and $\gamma: I \subseteq \mathbb{R} \rightarrow N$ is a non-null magnetic curve. Then $\gamma$ is a bi-f-harmonic curve iff the function $f$ and the constant scalar curvature requals to:

$$
f(s)=\text { constant }
$$

and

$$
r=\frac{2}{\eta(V)^{2}-\varepsilon_{1}}
$$

where $s \in I$.

Case III: If $\alpha=0$, that is to say $N$ is a $\beta$-para-Sasakian manifold, then equation (6.2) becomes:

$$
\left\{\begin{array}{l}
\left(f f^{\prime \prime}\right)^{\prime}+\left(2 f^{2}\right)^{\prime}(1-\beta \eta(V))=0, \\
3 f f^{\prime \prime}+2\left(f^{\prime}\right)^{2}+f^{2}\left(3 \beta^{2}(\eta(V))^{2}-2 \beta \eta(V)-\varepsilon_{1} \beta^{2}+1+\frac{r}{2}\left((\eta(V))^{2}-\varepsilon_{1}\right)\right)=0, \\
4 f f^{\prime}\left(\varepsilon_{1} \beta-\eta(V)\right)=0 .
\end{array}\right.
$$

We have the following corollaries by using third equation of Case III;

$$
\text { If } f^{\prime}=0 \text { we get; }
$$

Corollary 6.4. Let $N$ be a $\beta$-para-Sasakian manifold and $\gamma: I \subseteq \mathbb{R} \rightarrow N$ is a non-null magnetic slant curve. Then $\gamma$ is a bi-f-harmonic curve iff the function $f$ and the constant scalar curvature $r$ equals to:

$$
f(s)=\text { constant }
$$

and

$$
r=\frac{2\left(2 \beta \eta(V)-3 \beta^{2}(\eta(V))^{2}+\varepsilon_{1} \beta^{2}\right)}{(\eta(V))^{2}-\varepsilon_{1}}
$$

where $s \in I$.

$$
\text { If } \varepsilon_{1} \beta-\eta(V)=0 \text { we get; }
$$

Corollary 6.5. Let $N$ be a $\beta$-para-Sasakian manifold and $\gamma: I \subseteq \mathbb{R} \rightarrow N$ is a non-null magnetic slant curve. Then $\gamma$ is a bi-f-harmonic curve iff the function $f$ and the constant scalar curvature $r$ equals to:

$$
f(s)=c_{1} e^{\sqrt{2\left(\beta^{2} \varepsilon_{1}-1\right)} s}+c_{2} e^{-\sqrt{2\left(\beta^{2} \varepsilon_{1}-1\right)} s}
$$

and

$$
r=\frac{14 \beta^{2} \varepsilon_{1}+6 \beta^{4}-20}{\varepsilon_{1}-\beta^{2}}
$$

where $s \in I$ and $\varepsilon_{1} \beta-\eta(V)=0$.

If $f^{\prime}=0$ and $\varepsilon_{1} \beta-\eta(V)=0$ we get; 
Corollary 6.6. Let $N$ be a $\beta$-para-Sasakian manifold and $\gamma: I \subseteq \mathbb{R} \rightarrow N$ is a non-null magnetic slant curve. Then $\gamma$ is a bi-f-harmonic curve iff the function $f$ and the constant scalar curvature requals to:

$$
f(s)=\text { constant }
$$

and

$$
r=\frac{6 \beta^{4}-6 \beta^{2} \varepsilon_{1}-2}{\varepsilon_{1}-\beta^{2}}
$$

where $s \in I$ and $\varepsilon_{1} \beta-\eta(V)=0$.

Case IV: If $\eta(V)=\beta=0$, that is to say $N$ is a $\alpha$-para-Kenmotsu manifold and $\gamma$ is a Legendre curve, then equation (6.2) becomes:

$$
\left\{\begin{array}{l}
\left(f f^{\prime \prime}\right)^{\prime}+\left(2 f^{2}\right)^{\prime}=0 \\
3 f f^{\prime \prime}+2\left(f^{\prime}\right)^{2}+f^{2}\left(1-3 \alpha^{2} \varepsilon_{1}-\frac{r}{2} \varepsilon_{1}\right)=0 \\
f^{2} \varepsilon_{1} \alpha=0
\end{array}\right.
$$

We have the following corollary from Case IV;

Corollary 6.7. Let $N$ be a paracosymplectic manifold and $\gamma: I \subseteq \mathbb{R} \rightarrow N$ is a non-null magnetic Legendre curve. Then $\gamma$ is a bi-f-harmonic curve iff the function $f$ and the constant scalar curvature $r$ equals to:

$$
f(s)=c_{1} \cos (\sqrt{2} s)+c_{2} \sin (\sqrt{2} s)
$$

and

$$
r=\frac{4\left(f^{\prime}\right)^{2}-10 f^{2}}{\varepsilon_{1} f^{2}}
$$

where $s \in I$. (Here in Corollary 6.7, the constant of integration $c$ is taken as 0.)

Case V: If $\eta(V)=\alpha=0$, that is to say $N$ is a $\beta$-para-Sasakian manifold and $\gamma$ is a Legendre curve, then equation (6.2) becomes:

$$
\left\{\begin{array}{l}
\left(f f^{\prime \prime}\right)^{\prime}+\left(2 f^{2}\right)^{\prime}=0 \\
3 f f^{\prime \prime}+2\left(f^{\prime}\right)^{2}+f^{2}\left(1-\beta^{2} \varepsilon_{1}-\frac{r}{2} \varepsilon_{1}\right)=0 \\
4 f f^{\prime} \beta=0
\end{array}\right.
$$

We have the following corollaries from Case V;

Corollary 6.8. Let $N$ be a $\beta$-para-Sasakian manifold and $\gamma: I \subseteq \mathbb{R} \rightarrow N$ is a non-null magnetic Legendre curve. Then $\gamma$ is a bi-f-harmonic curve iff the function $f$ and the constant scalar curvature $r$ equals to:

$$
f(s)=\text { constant }
$$

and

$$
r=2\left(\varepsilon_{1}-\beta^{2}\right)
$$

where $s \in I$.

Corollary 6.9. Let $N$ be a paracosymplectic manifold and $\gamma: I \subseteq \mathbb{R} \rightarrow N$ is a non-null magnetic Legendre curve. Then $\gamma$ is a bi-f-harmonic curve iff the function $f$ and the constant scalar curvature $r$ equals to:

$$
f(s)=\text { constant }
$$

and

$$
r=2 \varepsilon_{1}
$$

where $s \in I$. 
Case VI: If $\beta=\alpha=0$, that is to say $N$ is a paracosymplectic manifold, then equation (6.2) becomes:

$$
\left\{\begin{array}{l}
\left(f f^{\prime \prime}\right)^{\prime}+\left(2 f^{2}\right)^{\prime}=0 \\
3 f f^{\prime \prime}+2\left(f^{\prime}\right)^{2}+f^{2}\left(1+\frac{r}{2}\left((\eta(V))^{2}-\varepsilon_{1}\right)\right)=0 \\
f f^{\prime} \eta(V)=0
\end{array}\right.
$$

We have the following corollary from Case VI;

Corollary 6.10. Let $N$ be a paracosymplectic manifold and $\gamma: I \subseteq \mathbb{R} \rightarrow N$ is a non-null magnetic curve. Then $\gamma$ is a bi-f-harmonic curve iff the function $f$ and the constant scalar curvature r equals to:

$$
f(s)=\text { constant }
$$

and

$$
r=\frac{2}{\varepsilon_{1}-(\eta(V))^{2}}
$$

where $s \in I$.

\section{7. f-Biminimal Non-Null Magnetic Curves}

Finally, in this section we derive the f-biminimality condition for a non-null magnetic curve in a threedimensional normal almost paracontact metric manifold and discuss the particular cases of paracosymplectic, $\alpha$-para-Kenmotsu and $\beta$-para-Sasakian manifolds.

We find the f-biminimality condition as below by using normal components of f-tension and f-bitension field with the help of $\lambda$-f-bienergy functional;

$$
\begin{aligned}
{\left[\tau_{2, \lambda, f}(\gamma)\right]^{\perp}=} & {\left[\tau_{2, f}(\gamma)\right]^{\perp}-\lambda\left[\tau_{f}(\gamma)\right]^{\perp} } \\
= & {\left[\left(1-\frac{r}{2}\left((\eta(V))^{2}-\varepsilon_{1}\right)-2 \beta \eta(V)+\left(\alpha^{2}+3 \beta^{2}\right)\left(\varepsilon_{1}-(\eta(V))^{2}\right)-\lambda\right) f-2 \alpha \eta(V) f^{\prime}+f^{\prime \prime}\right] \varphi V } \\
& +\left[\left(3 \alpha(\eta(V))^{2}-2 \varepsilon_{1} \alpha \beta \eta(V)-\varepsilon_{1} \alpha\right) f+2\left(\varepsilon_{1} \beta-\eta(V)\right) f^{\prime}\right] \xi \\
= & 0 .
\end{aligned}
$$

By using (7.1) we obtain;

Theorem 7.1. Let $N$ be a three-dimensional normal almost paracontact metric manifold with $\alpha, \beta=$ constant and $\gamma: I \subseteq \mathbb{R} \rightarrow N$ be a non-null magnetic curve. Then $\gamma$ is a f-biminimal curve iff the followings holds:

$$
\left\{\begin{array}{l}
\left(1-\frac{r}{2}\left((\eta(V))^{2}-\varepsilon_{1}\right)-2 \beta \eta(V)+\left(\alpha^{2}+3 \beta^{2}\right)\left(\varepsilon_{1}-(\eta(V))^{2}\right)-\lambda\right) f-2 \alpha \eta(V) f^{\prime}+f^{\prime \prime}=0, \\
\left(3 \alpha(\eta(V))^{2}-2 \varepsilon_{1} \alpha \beta \eta(V)-\varepsilon_{1} \alpha\right) f+2\left(\varepsilon_{1} \beta-\eta(V)\right) f^{\prime}=0 .
\end{array}\right.
$$

From Theorem 7.1, we deduce;

Corollary 7.1. Let $N$ be a three-dimensional normal almost paracontact metric manifold with $\alpha, \beta=$ constant and $\gamma: I \subseteq \mathbb{R} \rightarrow N$ be a non-null magnetic curve. Then $\gamma$ is a f-biminimal curve iff the function $f$ and the constant scalar curvature $r$ equals to:

$$
f(s)=e^{\left(\alpha \eta(V)+\frac{\alpha\left((\eta(V))^{2}-\varepsilon_{1}\right)}{2\left(\eta(V)-\varepsilon_{1} \beta\right)}\right) s+c}
$$

and

$$
r=\frac{2\left(\left(\frac{\alpha\left((\eta(V))^{2}-\varepsilon_{1}\right)}{2\left(\eta(V)-\varepsilon_{1} \beta\right)}\right)^{2}-(\eta(V))^{2}\left(3 \beta^{2}+2 \alpha^{2}\right)+\varepsilon_{1}\left(3 \beta^{2}+\alpha^{2}\right)-2 \beta \eta(V)+1-\lambda\right)}{(\eta(V))^{2}-\varepsilon_{1}}
$$

where $s \in I$. 
Case I: If $\eta(V)=0$, that is to say $\gamma$ is a Legendre curve, then equation (7.2) becomes:

$$
\left\{\begin{array}{l}
\left(\varepsilon_{1}\left(\beta^{2}-\alpha^{2}\right)+1+\frac{r}{2} \varepsilon_{1}+2 \varepsilon_{1}\left(\beta^{2}+\alpha^{2}\right)-\lambda\right) f+f^{\prime \prime}=0, \\
2 \beta f^{\prime}-\alpha f=0 .
\end{array}\right.
$$

We have the following corollary from Case I;

Corollary 7.2. Let $N$ be a three-dimensional normal almost paracontact metric manifold with $\alpha, \beta=$ constant and $\gamma: I \subseteq \mathbb{R} \rightarrow N$ be a non-null magnetic Legendre curve. Then $\gamma$ is a $f$-biminimal curve iff the function $f$ and the constant scalar curvature requals to:

$$
f(s)=e^{\frac{\alpha}{2 \beta} s+c}
$$

and

where $s \in I$.

$$
r=\left(2 \lambda-2-\frac{\alpha^{2}}{2 \beta^{2}}\right) \varepsilon_{1}-6 \beta^{2}-2 \alpha^{2}
$$

Case II: If $\alpha=0$, that is to say $N$ is a $\beta$-para-Sasakian manifold, then equation (7.2) becomes:

$$
\left\{\begin{array}{l}
\left(-2 \beta \eta(V)+1-\frac{r}{2}\left((\eta(V))^{2}-\varepsilon_{1}\right)-\beta^{2}\left(3(\eta(V))^{2}-3 \varepsilon_{1}\right)-\lambda\right) f+f^{\prime \prime}=0, \\
\left(\varepsilon_{1} \beta-\eta(V)\right) f^{\prime}=0 .
\end{array}\right.
$$

We have the following corollaries from Case II;

Corollary 7.3. Let $N$ be a $\beta$-para-Sasakian manifold and $\gamma: I \subseteq \mathbb{R} \rightarrow N$ be a non-null magnetic curve. Then $\gamma$ is a biminimal curve iff the constant scalar curvature $r$ equals to:

$$
r=\frac{2\left(3 \beta^{2} \varepsilon_{1}+1-3 \beta^{2}(\eta(V))^{2}-2 \beta \eta(V)-\lambda\right)}{(\eta(V))^{2}-\varepsilon_{1}} .
$$

Corollary 7.4. Let $N$ be a $\beta$-para-Sasakian manifold and $\gamma: I \subseteq \mathbb{R} \rightarrow N$ be a non-null magnetic slant curve. Then $\gamma$ is a biminimal curve iff the constant scalar curvature $r$ equals to:

$$
r=\frac{2\left(\beta^{2} \varepsilon_{1}+1-3 \beta^{4}-\lambda\right)}{\beta^{2}-\varepsilon_{1}} .
$$

Corollary 7.5. Let $N$ be a $\beta$-para-Sasakian manifold and $\gamma: I \subseteq \mathbb{R} \rightarrow N$ be a non-null magnetic slant curve. Then $\gamma$ is a f-biminimal curve iff the function $f$ and the constant scalar curvature $r$ are the solution of the following differential equation:

$$
\left.\left(1-\frac{r}{2}\left((\eta(V))^{2}-\varepsilon_{1}\right)-\eta(V)\right)^{2}\left(\varepsilon_{1}-3(\eta(V))^{2}\right)-\lambda\right) f+f^{\prime \prime}=0 .
$$

Case III: If $\beta=0$, that is to say $N$ is a $\alpha$-para-Kenmotsu manifold, then equation (7.2) becomes:

$$
\left\{\begin{array}{l}
\left(-\alpha^{2} \varepsilon_{1}+1-\frac{r}{2}\left((\eta(V))^{2}-\varepsilon_{1}\right)-\alpha^{2}\left((\eta(V))^{2}-2 \varepsilon_{1}\right)-\lambda\right) f-2 \alpha \eta(V) f^{\prime}+f^{\prime \prime}=0, \\
\left(3 \alpha(\eta(V))^{2}-\varepsilon_{1} \alpha\right) f-2 \eta(V) f^{\prime}=0 .
\end{array}\right.
$$

We have the following corollary from Case III;

Corollary 7.6. Let $N$ be a $\alpha$-para-Kenmotsu manifold and $\gamma: I \subseteq \mathbb{R} \rightarrow N$ be a non-null magnetic curve. Then $\gamma$ is a $f$-biminimal curve iff the function $f$ and the constant scalar curvature $r$ equals to:

$$
f(s)=e^{\left(\frac{3 \alpha(\eta(V))^{2}-\varepsilon_{1} \alpha}{2 \eta(V)}\right) s+c}
$$

and

$$
r=\frac{\left.2\left(-4(\eta(V))^{2} \alpha^{2}+2 \varepsilon_{1} \alpha^{2}+1-\lambda+\left(\frac{3 \alpha(\eta(V))^{2}-\varepsilon_{1} \alpha}{2 \eta(V)}\right)^{2}\right)\right)}{(\eta(V))^{2}-\varepsilon_{1}}
$$

where $s \in I$. 
Case IV: If $\eta(V)=\beta=0$, that is to say $N$ is a $\alpha$-para-Kenmotsu manifold and $\gamma$ is a Legendre curve, then equation (7.2) becomes:

$$
\left\{\begin{array}{l}
\left(1+\frac{r}{2} \varepsilon_{1}+\alpha^{2} \varepsilon_{1}-\lambda\right) f+f^{\prime \prime}=0, \\
\alpha f=0 .
\end{array}\right.
$$

We have the following corollary from Case IV;

Corollary 7.7. Let $N$ be a paracosymplectic manifold and $\gamma: I \subseteq \mathbb{R} \rightarrow N$ be a non-null magnetic Legendre curve. Then $\gamma$ is a f-biminimal curve iff the function $f$ equals to:

$$
f(s)=c_{1} \cos \left(\sqrt{1+\frac{r}{2} \varepsilon_{1}-\lambda} s\right)+c_{2} \sin \left(\sqrt{1+\frac{r}{2} \varepsilon_{1}-\lambda} s\right)
$$

where $s \in I ; c_{1}, c_{2} \in \mathbb{R}$ and $r$ is the constant scalar curvature.

Case V: If $\eta(V)=\alpha=0$, that is to say $N$ is a $\beta$-para-Sasakian manifold and $\gamma$ is a Legendre curve, then equation (7.2) becomes:

$$
\left\{\begin{array}{l}
\left(3 \varepsilon_{1} \beta^{2}+\frac{r}{2} \varepsilon_{1}-1-\lambda\right) f+f^{\prime \prime}=0, \\
\beta f^{\prime}=0 .
\end{array}\right.
$$

We have the following corollaries from Case V;

Corollary 7.8. Let $N$ be a $\beta$-para-Sasakian manifold and $\gamma: I \subseteq \mathbb{R} \rightarrow N$ be a non-null magnetic Legendre curve. Then $\gamma$ is a biminimal curve iff the constant scalar curvature $r$ equals to:

$$
r=2 \varepsilon_{1}\left(\lambda-1-3 \varepsilon_{1} \beta^{2}\right) .
$$

Corollary 7.9. Let $N$ be a paracosymplectic manifold and $\gamma: I \subseteq \mathbb{R} \rightarrow N$ be a non-null magnetic Legendre curve. Then $\gamma$ is a biminimal curve iff the constant scalar curvature $r$ equals to:

$$
r=2 \varepsilon_{1}(\lambda-1) .
$$

Case VI: If $\beta=\alpha=0$, that is to say $N$ is a paracosymplectic manifold, then equation (7.2) becomes:

$$
\left\{\begin{array}{l}
\left(1-\frac{r}{2}\left((\eta(V))^{2}-\varepsilon_{1}\right)-\lambda\right) f+f^{\prime \prime}=0, \\
\eta(V) f^{\prime}=0 .
\end{array}\right.
$$

We have the following corollary from Case VI;

Corollary 7.10. Let $N$ be a paracosymplectic manifold and $\gamma: I \subseteq \mathbb{R} \rightarrow N$ be a non-null magnetic curve. Then $\gamma$ is a biminimal curve iff the constant scalar curvature r equals to:

$$
r=\frac{2(1-\lambda)}{(\eta(V))^{2}-\varepsilon_{1}} .
$$

\section{Conclusion}

In this paper, we handled f-biharmonic and bi-f-harmonic non-null magnetic curves in three-dimensional normal almost paracontact metric manifolds. Because of the importance of these curves in physics and application fields of physics, we belive in that the paper has potential for further research. Thanks to the reviewers for their valuable comments. 


\section{References}

[1] Ara, M.: Geometry of f-harmonic maps. Kodai Mathematical Journal. 22, 243-263 (1999).

[2] Baird, P., Wood, JC.: Harmonic morphisms between Riemannian manifolds. London Mathematical Society Monographs. 29, Oxford Univ.Press. (2003).

[3] Caddeo, R.: Riemannian manifolds on which the distant function is biharmonic. Rendiconti del Seminario Matematico Universita Politecico Torino. 40, 93-101 (1982). https://doi.org/10.1515/math-2019-0112

[4] Calin, C., Crasmareanu, M.: Magnetic curves in three-dimensional quasi-para-Sasakian geometry. Mediterranean Journal of Mathematics. 13, 2087-2097 (2016). https:/ / doi.org/10.1007/s00009-015-0570-y

[5] Calvaruso, G, Munteanu, M.I., Perrone, A.: Killing magnetic curves in three-dimensional almost paracontact manifolds. Journal of Mathematical Analysis and Applications 426(1), 423-439 (2015). https:/ / doi.org/10.1016/j.jmaa.2015.01.057

[6] Chang, S.Y.A., Wang, L., Yang, P.C.: A regularity theory of biharmonic maps. Communications on Pure and Applied Mathematics, 52, 1113-1137 (1999). https:/ / doi.org/10.1002/(SICI)1097-0312(199909)52:91113::AID-CPA43.0.CO;2-7

[7] Course, N.: f-harmonic maps, PhD Thesis, University of Warwick, Coventry, UK, (2004).

[8] Dacko, P.: On almost para-cosymplectic manifolds. Tsukuba Journal of Mathematics. 28(1), 193-213 (2004).

[9] Druta-Romaniuc, S.L., Munteanu, M.I.: Killing magnetic curves in a Minkowski 3-space. Nonlinear Analysis: Real World Applications. 14(1), 383-396 (2013). https:/ /doi:10.1016/j.nonrwa.2012.07.002

[10] Druta-Romaniuc, S.L., Munteanu, M.I.: Magnetic curves corresponding to Killing magnetic fields in $E^{3}$. Journal of Mathematical Physics. 52(11), 1-14 (2011). https://doi.org/10.1063/1.3659498

[11] Eells, J., Sampson, J.H.: Harmonic mappings of Riemannian manifolds. American Journal of Mathematics. 86, 109-160 (1964).

[12] Eells, J., Lemaire, L.: A report on harmonic maps. Bulletin of the London Mathematical Society. 10, 1-68 (1978).

[13] Gürler, F., Özgür, C.: f-Biminimal immersions. Turkish Journal of Mathematics. 41, 564-575 (2017). https://doi:10.3906/mat-1508-23

[14] Jianguo, S.: The equations and characteristics of the Magnetic Curves in the Sphere Space. Advances in Mathematical Physics. Article ID 7694306.(2019). https://doi.org/10.1155/2019/7694306

[15] Kaneyuki S., Willams F.L.: Almost paracontact and paraHodge structures on manifolds. Nagoya Mathematical Journal. 99, 173-187 (1985).

[16] Keleş, S., Perktaş, S.Y., K1lıç, E.: Biharmonic Curves in LP-Sasakian Manifolds. Bulletin of the Malaysian Mathematical Sciences Society. 33(2), 325-344 (2010). https://doi.org/10.1155/2019/7694306

[17] Lu, W.J.: Onf-biharmonic maps and bi-f-harmonic maps between Riemannian manifolds. Science China Mathematics. 58, 1483-1498 (2015).

[18] Lu, W.J.: On f-biharmonic maps between Riemannian manifolds. Preprint arXiv:1305.5478 (2013).

[19] Loubeau, E., Montaldo, S.: Biminimal immersions. Proceedings of the Edinburgh Mathematical Society, 51, 421-437 (2008). https://doi.org/10.1017/S0013091506000393

[20] Montaldo, S., Oniciuc, C.: A short survey on biharmonic maps between Riemannian manifolds. Revista de la Union Matematica Argentina. 47(2), 1-22 (2006).

[21] Ou, Y.L.: Some constructions of biharmonic maps and Chen's conjecture on biharmonic hypersurfaces. Journal of Geometry and Physics, 62, 751-762 (2012). https://doi.org/10.1016/j.geomphys.2011.12.014

[22] Ou, Y.L.: On f-biharmonic maps and f-biharmonic submanifolds. Pacific Journal of Mathematics. 271(2), $461-477$ (2014).

[23] Ouakkas, S., Nasri, R., Djaa, M.: On the f-harmonic and f-biharmonic maps. JP Journal of Geometry and Topology. 10, 11-27 (2010).

[24] Perktaş, S.Y., Blaga, A.M., Acet, B.E., Erdoğan F.E.:Magnetic biharmonic curves on 3-dimensional normal almost paracontact metric manifolds. AIP Conference Proceedings. 020004,(2018). https://doi.org/10.1063/1.5047877

[25] Perktaş, S.Y., Blaga, A.M., Erdoğan, F.E., Acet, B.E.: Bi-f-Harmonic Curves and Hypersurfaces. Filomat. 33(16), 5167-5180 (2019). https://doi.org/10.2298/FIL1916167P

[26] Perktaş, S.Y., Kılıç, E.: Biharmonic Maps between Doubly Warped Product Manifolds. Balkan Journal of Geometry and its Applications. 15(2), $159-170$ (2010).

[27] Roth, J., Upadhyay, A.: f-biharmonic and bi-f-harmonic submanifolds of generalized space forms. Preprint arXiv. 1609.08599 (2016).

[28] Sario, L., Nakai, M., Wang, C., Chung, L.: Classification theory of Riemannian manifolds, Harmonic, quasiharmonic and biharmonic function. Lecture Notes in Mathematic 605, Springer-Verlag, Berlin-New York, (1977).

[29] Strzelecki, P.: On biharmonic maps and their generalizations. Calculus of Variations and Partial Differential Equations. 18, 401-432 (2003). https://doi: 10.1007/s100970200043.6.

[30] Wang, C.: Remarks on biharmonic maps into spheres. Calculus of Variations and Partial Differential Equations. 21, 221-242 (2004). https://doi.org/10.1007/s00526-003-0252-7

[31] Welyczko, J.: Slant curves in 3-dimensional normal almost paracontact metric manifolds. Mediterranean Journal of Mathematics. 11(3), 965-978 (2014). https:/ / doi:10.1007/s00009-013-0361-20378-620X/14/030965-14

[32] Welyczko, J.: On Legendre curves in 3-dimensional normal almost paracontact metric manifolds. Results in Mathematics. 54(34), 377-387 (2009).

[33] Zamkovoy, S.: Canonical connection on paracontact manifolds. Annals of Global Analysis and Geometry. 36, 37-60 (2009) https://doi.org/10.1007/s10455-008-9147-3

[34] Zhao, C.L., Lu, W.J.: Bi-f-harmonic map equations on singly warped product manifolds. Applied Mathematics-A Journal of Chinese Universities. 30(1), 111-126 (2015) https://doi:10.1007/s11766-015-3258-y 


\section{Affiliations}

ŞERIFE NUR BOZDAĞ

ADDRESS: Ege University, Dept. of Mathematics, 35040, İzmir-Turkey. E-MAIL: serife.nur.yalcin@ege.edu.tr ORCID ID:0000-0002-9651-7834

FEYZA ESRA ERDOĞAN

ADDRESS: Ege University, Dept. of Mathematics, 35040, İzmir-Turkey. E-MAIL: feyza.esra.erdogan@ege.edu.tr

ORCID ID:0000-0003-0568-7510 